\title{
Imaging isotopic content at the nanoscale using extreme ultraviolet laser ablation and ionization mass spectrometry
}

Lydia Rush, John Cliff, Dallas Reilly, Andrew Duffin, Carmen Menoni

Lydia A. Rush, John B. Cliff, Dallas D. Reilly, Andrew M. Duffin, Carmen S. Menoni, "Imaging isotopic content at the nanoscale using extreme ultraviolet laser ablation and ionization mass spectrometry," Proc. SPIE 11886, International Conference on X-Ray Lasers 2020, $118860 Z$ (8 July 2021); doi: $10.1117 / 12.2593315$

SPIE. Event: XVII International Conference on X-Ray Lasers, 2020, Online Only 


\title{
Imaging isotopic content at the nanoscale using extreme ultraviolet laser ablation and ionization mass spectrometry
}

\author{
Lydia A. Rush*a, John B. Cliff ${ }^{b}$, Dallas D. Reilly ${ }^{b}$, Andrew M. Duffin ${ }^{b}$, and Carmen S. Menoni ${ }^{a}$ \\ ${ }^{\mathrm{a}}$ Department of Electrical and Computer Engineering, Colorado State University, Fort Collins,

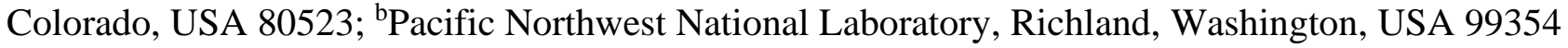

\begin{abstract}
Extreme ultraviolet (EUV) lasers possess unique properties for ablation and ionization at the nanoscale $(\leq 100 \mathrm{~nm})$ due to their short wavelength, high absorptivity in most materials (i.e., 10's of nanometers), and efficient photoionization in the laser-created plasmas. When coupled with a mass spectrometer, an EUV laser can be used to analyze and map chemical information in three dimensions with nanoscale spatial resolution. We have previously built an EUV time-of-flight mass spectrometer (EUV TOF) that achieved $\sim 80 \mathrm{~nm}$ lateral and $\sim 20 \mathrm{~nm}$ depth resolution when mapping the chemical content in organic and inorganic solids. Here, we present results from a recent study that extends EUV TOF's high resolution capabilities to the analysis of an isotopically heterogenous uranium fuel pellet that was made by blending two isotopically distinct starting materials. We show that EUV TOF can map ${ }^{235} \mathrm{U}^{238} \mathrm{U}$ heterogeneity at the $100 \mathrm{~nm}$ scale, revealing micron to submicron heterogeneity. For comparison, nanoscale secondary ionization mass spectrometry (NanoSIMS) maps a similar distribution of $U$ heterogeneity on a similar subsample at the same spatial scale. We also show that EUV TOF can measure the isotope ratio in a silver sample using single shot spectra. These results position EUV TOF as a promising technique for performing isotopic analyses at the nanoscale, finding applications in nuclear forensics, geology, and biology as well as in the semiconductor industry.
\end{abstract}

Keywords: Extreme Ultraviolet Lasers, Laser Ablation, Time-of-Flight Mass Spectrometry, Nanoscale, Isotopic Imaging and Mapping

\section{INTRODUCTION}

Mass spectral imaging with high spatial resolution is important in fields such as biology, ${ }^{1-3}$ geology,,${ }^{4}$ and nuclear forensics ${ }^{6-9}$ because it enables visualization of the spatial distribution of chemical/isotopic features of interest. Standard laser-based mass spectrometry methods, such as matrix assisted laser desorption and ionization (MALDI) and laser ablation inductively coupled plasma (LA ICP) mass spectrometry, are widely used for mapping molecular (MALDI MS), elemental, and isotopic (LA ICP-MS) information but are usually limited to analyses at the micron scale. ${ }^{1,7,10,11}$ Nanoscale secondary ionization mass spectrometry (NanoSIMS), an ion sputtering and ionization method, has become the leader in elemental and isotopic analyses at the nanoscale, achieving a lateral spatial resolution of <100 nm. . $^{3,5}$ Recently, we have shown that an extreme ultraviolet (EUV) laser coupled to a mass spectrometer can also offer unsurpassed three-dimensional (3D) nanoscale spatial resolution for chemical mapping. ${ }^{12-15}$

Our group at Colorado State University (CSU) has built and developed an EUV laser ablation and ionization time-offlight mass spectrometer (EUV TOF) that was the first of its kind to achieve mass spectral imaging with 3D nanoscale spatial resolution. ${ }^{12,13}$ The instrument uses a table-top EUV $\mathrm{Ar}^{8+}$ capillary discharge laser, also developed at CSU, that operates at a wavelength of $46.9 \mathrm{~nm}\left(26.4 \mathrm{eV}\right.$ photon energy). ${ }^{16,17}$ The $26.4 \mathrm{eV}$ photons are highly absorbed in mostly all materials, and the EUV laser beam can be focused to $80 \mathrm{~nm}$ diameters spots. ${ }^{18}$ This allows the EUV laser to ablate craters with volumes on the order of a few attoliters $\left(10^{-18} \mathrm{~L}\right)$ down to tens of zeptoliters $\left(10^{-21} \mathrm{~L}\right) .{ }^{12}$ Additionally, efficient EUV laser ionization takes place in the laser-created plasma plume. ${ }^{19,}{ }^{20}$ These unique properties of the EUV laser light allow for the analysis of nanoscale sized areas in a variety of solid materials, as we have shown that EUV TOF can accurately map molecular, elemental, and isotopic information with a lateral spatial resolution of $\sim 80 \mathrm{~nm}$ in organic and inorganic solids and with a depth resolution of $20 \mathrm{~nm}$ in organic materials. ${ }^{12,13}$

In a recent publication, we demonstrated EUV TOF's high spatial resolution capabilities to map isotopic heterogeneity in a uranium fuel pellet. ${ }^{14}$ More specifically, we showed that EUV TOF can map the ${ }^{235} \mathrm{U} /{ }^{238} \mathrm{U}$ isotope ratio in $100 \mathrm{~nm}$ sized 
pixels. The results revealed microscale (>100 $\mathrm{nm}$ ) heterogeneity with domains characteristic of the two starting materials (i.e., feedstock). EUV TOF mapped similar distributions of heterogeneity to NanoSIMS on a separate sub-sample prepared from the same material and also at the $\sim 100 \mathrm{~nm}$ spatial scale. While the isotopic results return a single distribution, indicating a decently mixed product, close analysis of both EUV TOF and NanoSIMS data revealed statistically significant (i.e., real) heterogeneity associated with spatial domains of less mixed feedstock. These results further highlighted EUV TOF's ability to accurately map isotopic content at the nanoscale by exposing the two distinct feedstocks. Other studies showed that the isotopic heterogeneity in this sample could not be fully revealed by microscale or bulk mass spectrometry techniques. ${ }^{7}$

Here, we compliment this recent study with additional analysis demonstrating the inability of microscale techniques to reveal the true sample heterogeneity. We show that EUV TOF mappings of the ${ }^{235} \mathrm{U} /{ }^{238} \mathrm{U}$ isotope ratio using $1 \mu \mathrm{m}$ pixels on the same heterogenous uranium fuel pellet mentioned above does not completely reveal the uranium fuel sample's heterogeneity. This result further confirms that the sample's microscale heterogeneity requires high spatial mapping for accurate identification. We also show results from a separate study that further showcases EUV TOF's capability for isotopic analyses by analyzing a pure, homogenous silver sample. Silver is isotopically ideal for isotope ratio analyses because it has equally abundant major isotopes $\left(\sim 50 \%{ }^{107} \mathrm{Ag}\right.$ and $\left.{ }^{109} \mathrm{Ag}\right)$. We show that EUV TOF can accurately measure the isotope ratio in silver from single shot mass spectra. These results show that EUV TOF is a promising tool towards applications that require isotope ratio analyses and/or mapping at high spatial scales in a variety of materials.

\section{EXPERIMENTAL SECTION}

The EUV TOF mass spectrometer, developed and built at CSU, has been described in detail elsewhere. ${ }^{12-14}$ In brief, the table-top Ne-like $\mathrm{Ar}^{8+}$ EUV capillary discharge laser ${ }^{16}$ (sized $0.4 \mathrm{~m}^{2}$ or $\sim 3 \mathrm{ft}^{2}$ ) operates at a wavelength of $46.9 \mathrm{~nm}$ with a maximum pulse energy of $\sim 10 \mu \mathrm{J}$ and duration of $\sim 1.5 \mathrm{~ns} .{ }^{17}$ The EUV laser beam illuminates a free-standing zone plate $(\mathrm{NA}=0.12)$ that focuses the beam onto the sample. ${ }^{12,21}$ The laser's fluence can be adjusted with an argon gas pressure cell to allow for more or less ablation at the sample. ${ }^{12}$ The sample is moved in nano- to micro-scale sized increments using piezoelectric stages (Physik Instrumente, Q-545.240), where the sample's step size determines the pixel size of the resulting ion maps. A set of ion optics is used to extract and accelerate the ions created in the EUV laser-created plasma plume into a field-free TOF mass spectrometer (Jordan TOF Products) operated in reflectron mode, where ions are separated in time according to their mass/charge $(\mathrm{m} / \mathrm{z})$ ratio over a $\sim 2$ meter distance. The ions are detected by a chevron-type microchannel plate (MCP) detector and digitized by an analog-to-digital converter (ADC) (Dynamic Signals, EON Compuscope CS121G1). The typical mass resolving power $(\mathrm{m} / \Delta \mathrm{m})$ for the EUV TOF is $\sim 1100 .{ }^{12,13}$

EUV TOF and NanoSIMS mapped the ${ }^{235} \mathrm{U} /{ }^{238} \mathrm{U}$ ratio in a heterogenous low enriched uranium (LEU, $\sim 2 \%{ }^{235} \mathrm{U}$ and $\sim 98 \%{ }^{238} \mathrm{U}$ ) fuel sample ${ }^{22}$ and natural uranium (NU, $0.725 \%{ }^{235} \mathrm{U}$ and $\sim 99 \%{ }^{238} \mathrm{U}$ ) metal certified reference material (NBL, CRM 112-A ${ }^{23}$ prepared at Pacific Northwest National Laboratory using a focused ion beam/scanning electron microscope (FIB/SEM). ${ }^{14}$ The sample dimensions were $\sim 20 \mu \mathrm{m}$ x $20 \mu \mathrm{m}$ x $3 \mu \mathrm{m}$ (Figure 1A). All samples were coated with conductive gold coating prior to analysis. Further details on sample preparation can be found elsewhere. ${ }^{7}{ }^{14}$ EUV TOF mapped the ${ }^{235} \mathrm{U}^{+},{ }^{238} \mathrm{U}^{+},{ }^{235} \mathrm{U}^{16} \mathrm{O}^{+}$, and ${ }^{238} \mathrm{U}^{16} \mathrm{O}^{+}$isotopes over the LEU and NU samples using $1 \mu \mathrm{m}$ pixels (i.e., sample step size) at a laser fluence of $\sim 1 \mathrm{~J} / \mathrm{cm}^{2}$ with mapping field sizes of $\sim 25 \mu \mathrm{m}$ x $25 \mu \mathrm{m}$ (Figure 1B,C). The same isotopes were re-mapped over a small $\sim 20 \mu \mathrm{m}$ x $1 \mu \mathrm{m}$ area of the LEU sample using $100 \mathrm{~nm}$ pixels at a laser fluence of $<1 \mathrm{~J} / \mathrm{cm}^{2}$ (Figure 1D) ${ }^{14}$ laser conditions previously used to ablate craters in resist with a diameter $\leq 400 \mathrm{~nm}$ and depth $\leq 40 \mathrm{~nm} .{ }^{12}$ NanoSIMS mapped the same isotope species on a similar LEU subsample using $98 \mathrm{~nm}$ pixels with a 2-4 pA $\mathrm{O}^{-}$primary ion beam with a diameter of $\sim 300 \mathrm{~nm}$ (Figure 1E). ${ }^{14}$ For both EUV TOF and NanoSIMS analyses, the ${ }^{235} \mathrm{U}$ and ${ }^{235} \mathrm{UO}$ ion counts and ${ }^{238} \mathrm{U}$ and ${ }^{238} \mathrm{UO}$ ions collected at each pixel (i.e., the background-subtracted peak area) were subjected to a threshold and summed to a single pixel. ${ }^{13,}{ }^{24}$ The threshold was used to remove insufficient ${ }^{235} \mathrm{U},{ }^{235} \mathrm{UO}$ signals (i.e., 0 ion counts of the minor isotope for EUV TOF and NanoSIMS analysis) or saturated ${ }^{238} \mathrm{U},{ }^{238} \mathrm{UO}$ signals (i.e., counts of the major isotope above the ADC's upper dynamic range for EUV TOF analysis). Three laser shots were taken at each pixel location for EUV TOF analyse ${ }^{13,25}$ and eight passes per pixel were collected with NanoSIMS. In this study the NU sample acted as a bracketing standard. Further information on EUV TOF and NanoSIMS U ratio analyses can be found in Rush et al. ${ }^{14}$ 
EUV TOF ${ }^{107} \mathrm{Ag} /{ }^{109} \mathrm{Ag}$ ratio analysis was performed using a $\geq 99 \%$ pure silver foil (GoodFellow, AG000467) with a natural $\mathrm{Ag}$ abundance (i.e., $\sim 52 \%{ }^{107} \mathrm{Ag}$ and $\sim 48 \%{ }^{109} \mathrm{Ag}$ ). Individual mass spectra were collected over a large area sized $500 \mu \mathrm{m} \times 500 \mu \mathrm{m}$ with $50 \mu \mathrm{m}$ steps between each ablation spot and six laser shots at every spot using a laser fluence of $\sim 1 \mathrm{~J} / \mathrm{cm}^{2}$. The ${ }^{107} \mathrm{Ag} /{ }^{109} \mathrm{Ag}$ isotope ratio was calculated from each background-subtracted peak area in the mass spectrum. Unlike the $\mathrm{U}$ mapping analysis above, spatial information was not important for this analysis because the Ag foil is expected to be homogenous throughout its entire area. Instead, the focus here was to further assess EUV TOF's capabilities for accurate isotopic analyses by analyzing single shot mass spectra using an "easy-to-work-with" sample, that is, a sample with major isotopes that are separated by a few mass units and that have similar abundances to avoid challenges associated with the instrument's dynamic range.

\section{RESULTS}

\section{a. EUV TOF ${ }^{235} \mathrm{U}^{238} \mathrm{U}$ isotope ratio maps of heterogenous and homogenous uranium samples}

EUV TOF mapped the ${ }^{235} \mathrm{U} /{ }^{238} \mathrm{U}$ isotope ratio across the heterogenous LEU and homogenous NU CRM FIB-fabricated samples at the micro- and nano-scales. ${ }^{14}$ Analysis of the NU CRM allows EUV TOF's instrumental response for assessing isotopic heterogeneity to be measured. Figure 1A shows an SEM image of one of the FIB samples prior to EUV TOF (or NanoSIMS) analysis. ${ }^{7}$ Figure 1B,C shows resulting EUV TOF ${ }^{235} \mathrm{U} /{ }^{238} \mathrm{U}$ maps across the entire LEU and NU CRM sample areas, respectively, using $1 \mu \mathrm{m}$ pixels. Figure 1D shows a subsection of the LEU pellet remapped using $100 \mathrm{~nm}$ pixels. ${ }^{14}$ For comparison to Figure 1D, Figure 1E shows a similarly sized NanoSIMS ratio map from a different FIB extracted subsample collected with $\sim 100 \mathrm{~nm}$ pixels. ${ }^{14}$ All maps are plotted on the same color scale ${ }^{235} \mathrm{U} /{ }^{238} \mathrm{U}=0.002$ to 0.050

A)

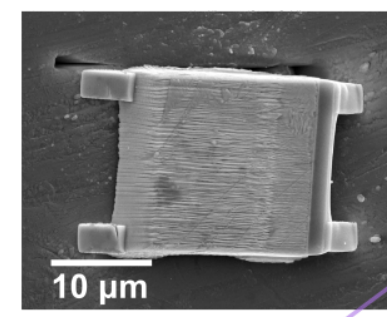

D)

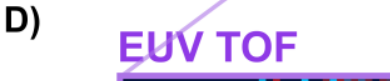

B)

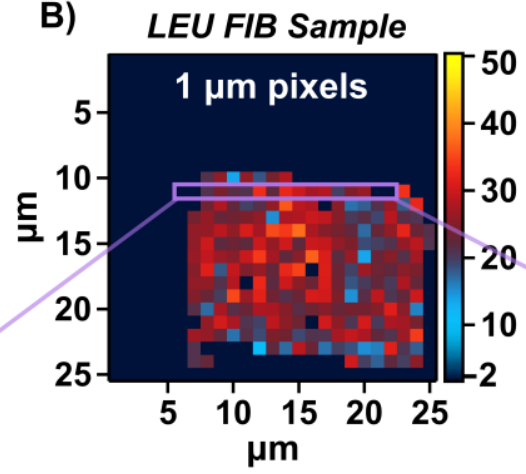

C)

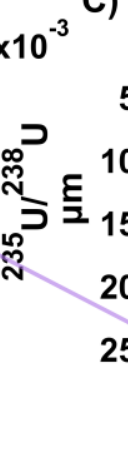

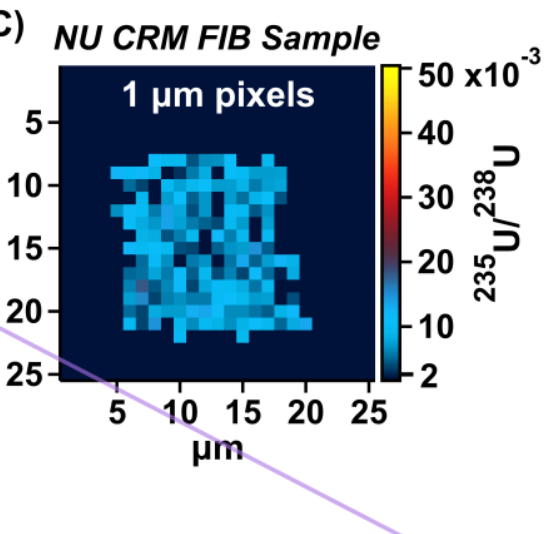

$100 \mathrm{~nm}$ pixels

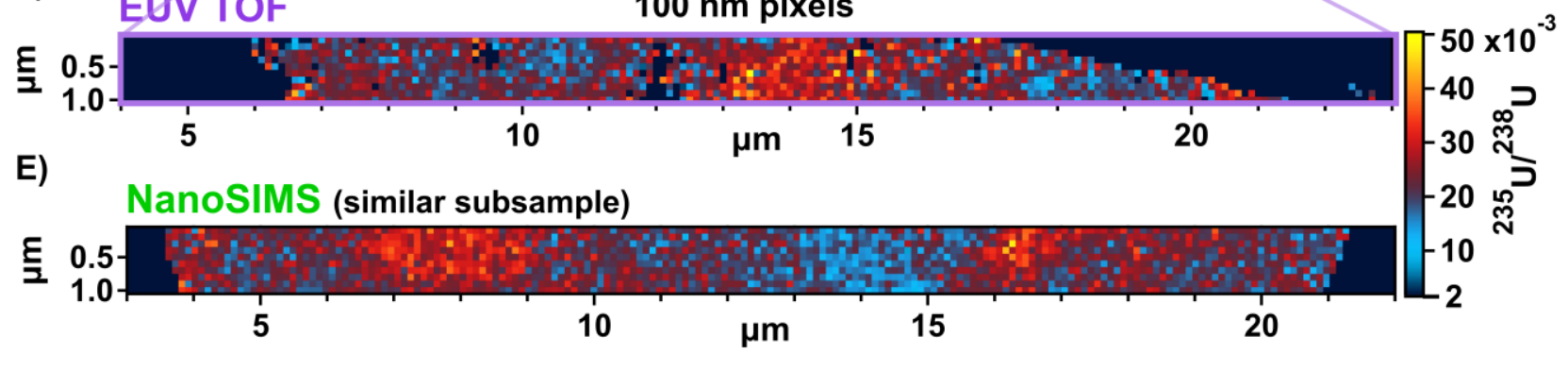

Figure 1. (A) SEM image of a uranium FIB-fabricated sample prior to analysis. (B-C) EUV TOF ${ }^{235} \mathrm{U} /{ }^{238} \mathrm{U}$ ratio maps using $1 \mu \mathrm{m}$ pixels (i.e., step size) of the (B) heterogenous LEU FIB sample and (C) homogenous NU CRM FIB sample, both with mapping field sizes of $25 \mu \mathrm{m} \times 25 \mu \mathrm{m}$. (D) EUV TOF ${ }^{235} \mathrm{U} /{ }^{238} \mathrm{U}$ ratio map of a $19 \mu \mathrm{m} \times 1 \mu \mathrm{m}$ section of the same LEU sample mapped in (B) using $100 \mathrm{~nm}$ pixels. (E) NanoSIMS ratio map of a different $19 \mu \mathrm{m} \times 1 \mu \mathrm{m}$ section of a LEU sample mapped with $98 \mathrm{~nm}$ pixels. The pixels in all ratio maps are not smoothed or interpolated. 
The EUV TOF ${ }^{235} \mathrm{U} /{ }^{238} \mathrm{U}$ ratio maps in Figure 1B,C show that the NU CRM clearly has a reasonably homogeneous $\mathrm{U}$ isotopic content throughout the entire sample area, with slight variations based on instrument limitations and counting statistics. On the other hand, the LEU sample has more U isotopic variations throughout the sample, which is evident by the $1 \mu \mathrm{m}$ areas of lower (blue) and higher (red) ${ }^{235} \mathrm{U} /{ }^{238} \mathrm{U}$ ratios. This indicates that the LEU sample, which was made by blending two isotopically distinct feedstocks, does have heterogeneity at the microscale. However, Figure 1D shows that when EUV TOF re-maps a small section of the LEU pellet with $100 \mathrm{~nm}$ pixels more ${ }^{235} \mathrm{U} /{ }^{238} \mathrm{U}$ variations are revealed in the sample that were not visible at the $1 \mu \mathrm{m}$ scale. While isotopic mixing of the starting materials does seem to be successful at the $100 \mathrm{~nm}$ spatial scale, there are $\sim 1-2 \mu \mathrm{m}$ sized patches of heterogeneity that are exposed across the length of the sample that indicate less or unmixed starting materials, likely micrometer sized oxide powders. ${ }^{6,}{ }^{14}$ For comparison, Figure 1E shows the resulting NanoSIMS ratio map that exposes similarly sized regions of heterogeneity at about the same spatial scale. This result is significant because it shows that EUV TOF has similar isotopic mapping capabilities to NanoSIMS.

It should be noted that EUV TOF and NanoSIMS maps in Figure 1 have not undergone any image post-processing such as smoothing or interpolation, which can be required when the pixel/step size is much larger than the beam's spot size or when a more visually appealing image or map is desired (at the cost of a loss in spatial resolution). The maps shown in Figure 1 are therefore significant because they show EUV TOF (and NanoSIMS) can map the ${ }^{235} U^{238} \mathrm{U}$ isotope ratio pixel by pixel in the uranium samples. ${ }^{13,14}$

\section{b. EUV TOF statistical analysis of the ${ }^{235} \mathrm{U} /{ }^{238} \mathrm{U}$ isotope ratio maps}

The EUV TOF and NanoSIMS ${ }^{235} \mathrm{U}^{238} \mathrm{U}$ ratio maps in Figure 1 provide a qualitative picture of the LEU sample's heterogeneity. Statistical analysis of the mapped data was subsequently performed to try and further resolve this heterogeneity. ${ }^{14}$ Figure 2A shows EUV TOF and NanoSIMS two-isotope plots of the LEU and NU CRM mapped data from Figure 1. The NanoSIMS data was taken from the entire $20 \mu \mathrm{m}$ x $20 \mu \mathrm{m}$ LEU FIB sample area, while the EUV TOF $100 \mathrm{~nm}$ data were taken from the smaller $19 \mu \mathrm{m} \times 1 \mu \mathrm{m}$ map in Figure 1D. This explains why there are more NanoSIMS data points in the plots in Figure 2. Figure 2B shows corresponding ${ }^{235} \mathrm{U} /{ }^{238} \mathrm{U}$ ratio plots, where the data are shown with $\pm 2 \sigma$ and $\pm 3 \sigma$ uncertainty envelopes at the respective ${ }^{238} \mathrm{U}$ ion counts (counting statistics uncertainty). The counting statistics uncertainty provides a measure of the instruments' variability based on the number of atoms detected and allows the identification of statistically significant points of heterogeneity. Error bars are not shown on the points in Figure 2B for clarity but would be the width of the $\pm 2 \sigma$ and $\pm 3 \sigma$ envelopes at the corresponding ion counts. Variations in measured ion intensities are likely from a combination of shot-to-shot laser energy fluctuations, slight variations in the sample (e.g. topography, geometry, and conductivity), and the probability of detecting infrequent ion events. Figure $2 \mathrm{C}$ plots the ratio data in Figure 2B as Gaussian-fitted histograms (made according to the Freedman-Diaconis rule). The histogram provides a metric for the width of the ${ }^{235} \mathrm{U}^{238} \mathrm{U}$ distributions measured with EUV TOF and NanoSIMS in each sample at the micro- and nano-scales. The value of the slope of the linear best fit lines (Figure 2A), expected/certified ratios (Figure 2A,B), and width of the ${ }^{235} \mathrm{U}^{238} \mathrm{U}$ distributions (Figure $2 \mathrm{C}$ ) is given in Table 1 .

Figure $2 \mathrm{~A}$ shows the linear best fit lines through the data, where the slope of the lines is the value of the average ratios, listed in Table 1. EUV TOF measures a ${ }^{235} \mathrm{U}^{238} \mathrm{U}=0.0072 \pm 0.0008(2 \sigma)$ in the NU CRM, where the certified ratio is $0.00725 .{ }^{23}$ Because the measured ratio agrees with the certified ratio within analytical error, there was no need to make mass bias corrections to the data. The average ratio of the $100 \mathrm{~nm}$ pixel analyses on the LEU sample with EUV TOF and NanoSIMS also agrees with the expected ratio from bulk measurements (Table 1). ${ }^{7}$ This result indicates that while each FIB'd subsample is expected to show unique variations, the $19 \mu \mathrm{m} \times 1 \mu \mathrm{m}$ area and $100 \mathrm{~nm}$ pixel sampling proved sufficient to approximate the bulk measurement while simultaneously exposing the heterogeneity. However, the average ${ }^{235} \mathrm{U}^{238} \mathrm{U}$ ratio of the $1 \mu \mathrm{m}$ pixel analysis on the LEU sample with EUV TOF is higher than the expected ratio. The map in Figure 1B also shows that higher ratio areas are more prevalent. During the $1 \mathrm{um}$ spot analysis it was likely that the laser spot size was smaller than the $1 \mu \mathrm{m}$ pixel size. This, in conjunction with the limited number of data points, caused the LEU sample to be under sampled resulting in an artificially high ratio. Along these same lines, the EUV TOF and NanoSIMS $100 \mathrm{~nm}$ pixel analyses also show a slightly higher (but overlapping) average ratio compared to the expected ratio for the LEU sample. This could be the result of comparing EUV TOF and NanoSIMS results from a single LEU sample to bulk measurements (i.e., the expected ratio) that were taken over multiple LEU samples, with each sample possessing slightly different $U$ variations.

Figure 2A,B show that all of the data points collected with EUV TOF on the NU CRM sample fall within $\pm 3 \sigma$ of the expected uncertainty, with the majority of points falling within $\pm 2 \sigma$. Additionally, the few points that fall outside of the $\pm 2 \sigma$ uncertainty are at low ${ }^{238} \mathrm{U}$ counts (i.e., $<1000$ counts). On the other hand, the LEU sample mapped with $1 \mu \mathrm{m}$ and 

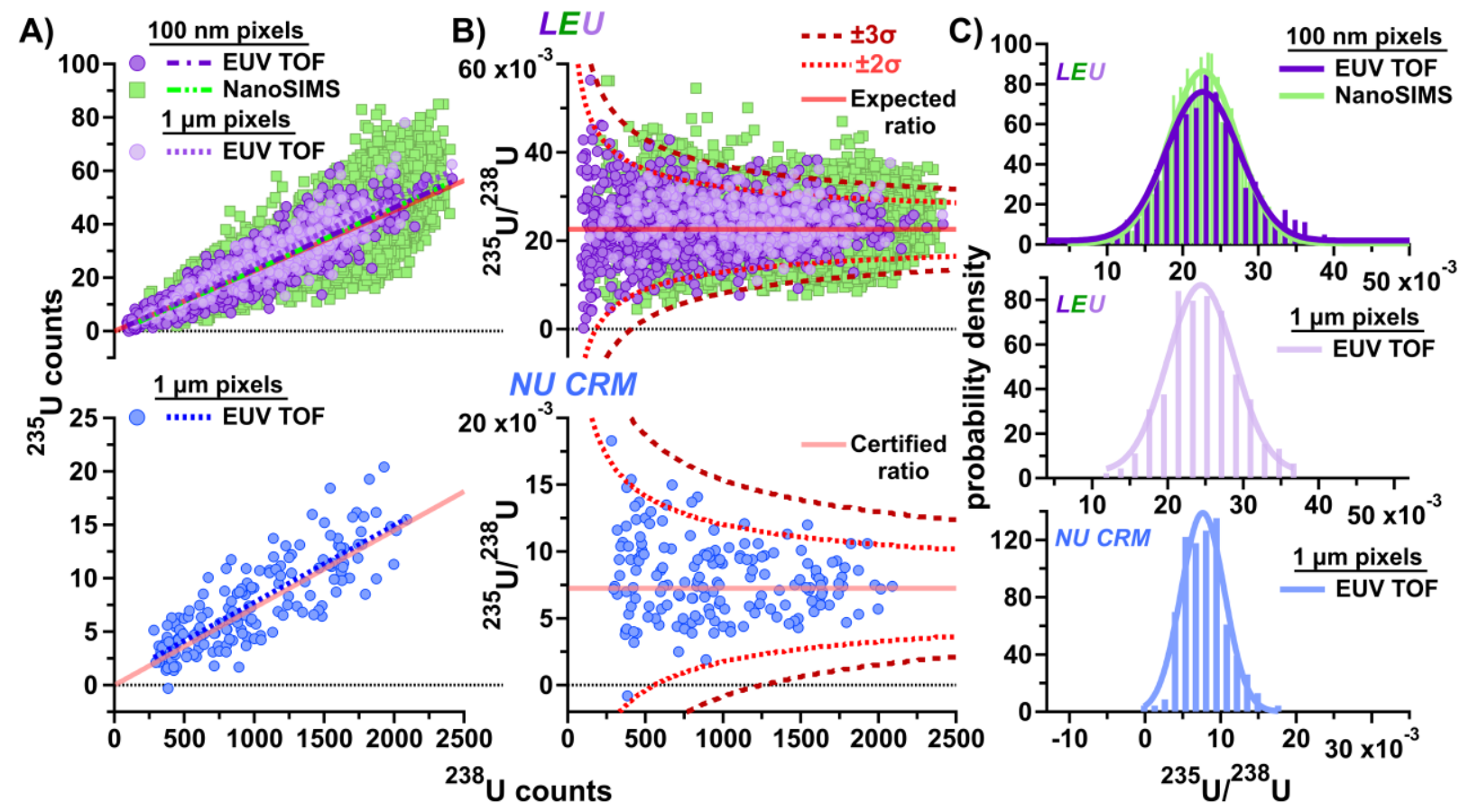

Figure 2. Uranium (A) two-isotope, (B) ratio, and (C) histogram plots of the EUV TOF and NanoSIMS data collected on the LEU (top plots) and NU CRM (bottom plots) samples at the micro- and nano-scales. The EUV TOF data shown in these plots is the same mapped data in Figure 1. (A) ${ }^{235} \mathrm{U}$ counts plotted as a function of ${ }^{238} \mathrm{U}$ counts for the analysis of the LEU and NU CRM samples with EUV TOF and NanoSIMS using $100 \mathrm{~nm}$ and $1 \mu \mathrm{m}$ pixels. All data sets are fitted with a dotted linear best fit line (intercepts for the measured data are not fixed at zero) that corresponds to the average ratio, whose value is given in Table 1. (B) Corresponding ${ }^{235} \mathrm{U} /{ }^{238} \mathrm{U}$ ratios plotted as a function of ${ }^{238} \mathrm{U}$ counts for the LEU and NU CRM samples. The red dotted lines represent the $\pm 2 \sigma$ and $\pm 3 \sigma$ error from the expected/certified ratio value, shown as a red solid line in (A,B), based on counting statistics. (C) Histogram plots of the probability density (normalized) as a function of the ${ }^{235} \mathrm{U} /{ }^{238} \mathrm{U}$ ratio plotted in (B) for the LEU sample using $100 \mathrm{~nm}$ pixels (top) and $1 \mu \mathrm{m}$ pixels (middle) and for the NU CRM using $1 \mu \mathrm{m}$ pixels (bottom). The width of each Gaussian-fitted histogram distribution is listed in Table 1.

Table 1. EUV TOF and NanoSIMS average ${ }^{235} \mathrm{U} /{ }^{238} \mathrm{U}$ ratios and the corresponding width of the ratio distributions from the analysis of the LEU and NU CRM samples. The ${ }^{235} \mathrm{U} /{ }^{238} \mathrm{U}$ ratios were calculated from the slope of the linear best fit lines in Figure $2 \mathrm{~A}$, and the corresponding ${ }^{235} \mathrm{U} /{ }^{238} \mathrm{U}$ distributions are the width of the Gaussian-fitted histograms in Figure $2 \mathrm{C}$. The shapes and lines in the table correspond to the data in Figure 2.

\begin{tabular}{|c|c|c|c|}
\hline \multicolumn{4}{|c|}{ LEU fuel pellet, ${ }^{235} \mathrm{U} /{ }^{238} \mathrm{U}\left(\right.$ expected $\left.^{\mathrm{a}}\right)=0.0226 \pm 0.0006(2 \sigma)$} \\
\hline Technique & Pixel size & Average ${ }^{235} U /{ }^{238} U \pm 2 \sigma$ error & Width of ${ }^{235} \mathrm{U} /{ }^{238} \mathrm{U}$ distribution \\
\hline EUV TOF & $100 \mathrm{~nm} \bigcirc$ & $0.0232 \pm 0.0006-\cdots$ & $0.055 \longrightarrow$ \\
\hline NanoSIMS & $100 \mathrm{~nm} \square$ & $0.0234 \pm 0.0002 \ldots$ & $0.051=$ \\
\hline EUV TOF & $1 \mu \mathrm{m} \bigcirc$ & $0.0250 \pm 0.0017 \ldots \ldots \ldots$ & $0.025=$ \\
\hline \multicolumn{4}{|c|}{ NU CRM $112-A,{ }^{235} U /{ }^{238} U$ (certified $)=0.00725$} \\
\hline Technique & Pixel size & Average ${ }^{235} \mathrm{U} /{ }^{238} \mathrm{U} \pm 2 \sigma$ error & Width of ${ }^{235} \mathrm{U} /{ }^{238} \mathrm{U}$ distribution \\
\hline EUV TOF & $1 \mu \mathrm{m} \bigcirc$ & $0.0072 \pm 0.0008 \ldots \ldots \ldots \ldots$ & $0.018=$ \\
\hline
\end{tabular}

aThe expected ratio is based on the aggregated ratio from NanoSIMS, large geometry (LG)SIMS, LA ICP-MS, quadrupole ICP-MS, and thermal ionization MS (TIMS) analyses on multiple pellets. ${ }^{7}$ 
$100 \mathrm{~nm}$ pixels with EUV TOF shows more points that fall outside the $\pm 2 \sigma$ uncertainty, with a few points falling outside $\pm 3 \sigma$ at ${ }^{238} \mathrm{U}$ counts $>1000$. Because the analysis of the $\mathrm{NU}$ reference material shows that the measured variability does not exceed what is expected based on the number of atoms detected, the ${ }^{235} \mathrm{U}^{238} \mathrm{U}$ ratios measured in the LEU sample that fall outside the $\pm 3 \sigma$ counting statistics uncertainty are likely from isotopic heterogeneity rather than instrumental uncertainty. Additionally, NanoSIMS analysis of the LEU sample confirms EUV TOF's measurements with overlapping $\mathrm{U}$ ratios detected outside of the counting statistics uncertainty. More NanoSIMS data points fall outside of the $\pm 3 \sigma$ envelope because, as mentioned above, data from the entire sample area were included for the NanoSIMS' plots in Figure 2.

Figure 2A,B also show the advantage of mapping the LEU sample at the nanoscale versus at the microscale. The EUV TOF data collected on the LEU sample using $1 \mu \mathrm{m}$ pixels shows evidence of statistically significant heterogeneity. However, EUV TOF's $100 \mathrm{~nm}$ pixel analysis shows more heterogeneity with more points falling outside the $\pm 3 \sigma$ uncertainty. This is further evidenced in the ${ }^{235} \mathrm{U} /{ }^{238} \mathrm{U}$ histogram distributions, where the width of the Gaussian-fitted distribution for the EUV TOF $100 \mathrm{~nm}$ pixel analysis is $\sim 2 \mathrm{x}$ larger than the $1 \mu \mathrm{m}$ pixel analysis collected on the LEU sample. The width of the EUV TOF and NanoSIMS ${ }^{235} \mathrm{U} /{ }^{238} \mathrm{U}$ distributions collected with $100 \mathrm{~nm}$ pixels on the LEU sample are $>0.05$, while the width of the ratio distribution collected with $1 \mu \mathrm{m}$ pixels is $\sim 0.03$ (Table 1 ). For comparison, the width of the ratio distribution of the NU CRM collected with EUV TOF is $\sim 0.02$ (Table 1). This finding further confirms that the LEU sample has microscale heterogeneity that requires high spatial mapping for identification.

\section{c. EUV TOF analysis of an isotopically ideal sample}

EUV TOF's capabilities to accurately determine isotope ratios were also tested by analyzing a pure silver foil. Silver has two major isotopes, ${ }^{107} \mathrm{Ag}$ and ${ }^{109} \mathrm{Ag}$, that are both $\sim 50 \%$ naturally abundant, unlike the LEU and NU samples that had a disproportionate abundance of ${ }^{238} \mathrm{U}(\geq 98 \%)$. The Ag sample is therefore an ideal scenario for the EUV TOF system in terms of dynamic range and sensitivity. Figure 3 shows the resulting two isotope and ${ }^{107} \mathrm{Ag} /{ }^{109} \mathrm{Ag}$ ratio plots from single shot spectra. The Ag ion counts are much lower than the $\mathrm{U}$ analysis above because each data point was obtained from an individual laser shot, whereas the $\mathrm{U}$ and UO signals from three laser shots per spot were summed to a single pixel/data point in Figures 1 and 2. Nonetheless, Figure 3 shows that even at low ion counts, EUV TOF can accurately determine the isotope ratio from individual ablation events. The average ratio measured from single shot EUV TOF spectra is ${ }^{107} \mathrm{Ag} /{ }^{109} \mathrm{Ag}=1.11 \pm 0.03(2 \sigma)$. The measured ratio is in close agreement with the certified value of 1.076 . While each point in Figure 3B has a large uncertainty, it is surprising that mostly all of the points fall within $\pm 2 \sigma$ of the certified value given the low ion count rate of $\leq 100$. It should also be noted that the ${ }^{107} \mathrm{Ag} /{ }^{109} \mathrm{Ag}$ distribution (i.e., the Gaussianfitted histogram) cannot be directly compared to the $U$ analysis since the former was performed at much lower ion counts where the distribution is expected to be larger based on counting statistics. Nonetheless, the Ag analysis shows that EUV TOF can determine the isotope ratio in a single laser shot in the case in which the isotopes of interest have a similar abundance and when the ion count rate is sufficient (i.e., individual ${ }^{107} \mathrm{Ag} /{ }^{109} \mathrm{Ag}$ ratios measured at $\sim 100$ ion counts all show good agreement with the certified value in Figure 3B). This could be beneficial for particle analyses, where the amount of material is limited.

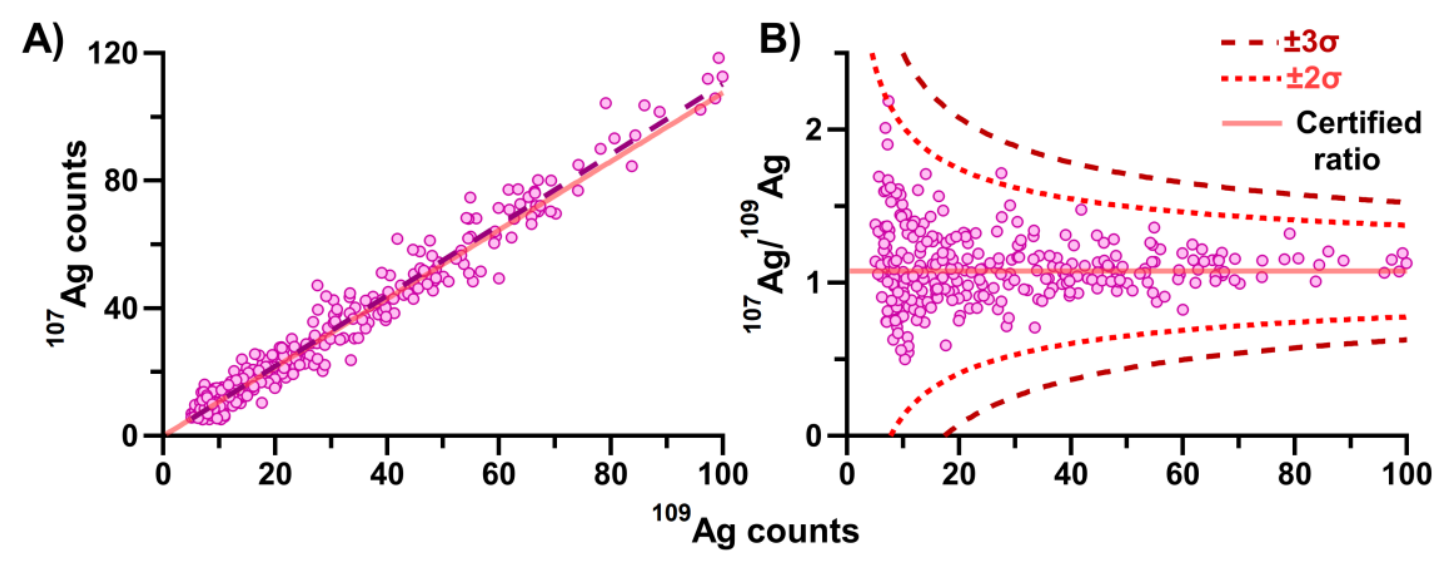

Figure 3. Silver (A) two-isotope and (B) ratio plots of the EUV TOF data collected on the homogenous silver foil. Each data point is from a single laser shot. (A) ${ }^{107} \mathrm{Ag}$ counts plotted as a function of ${ }^{109} \mathrm{Ag}$ counts. The dotted line represents the linear 
best fit line (average ratio) of the EUV TOF data (intercept of line is not fixed at zero), whose slope is $1.11 \pm 0.03(2 \sigma)$. The certified ${ }^{107} \mathrm{Ag} /{ }^{109} \mathrm{Ag}$ ratio, shown as a red solid line in $(\mathrm{A}, \mathrm{B})$, is 1.076 . (B) Corresponding ${ }^{107} \mathrm{Ag} /{ }^{109} \mathrm{Ag}$ ratios plotted as a function of ${ }^{109} \mathrm{Ag}$ counts. The red dotted lines represent the $\pm 2 \sigma$ and $\pm 3 \sigma$ error from the certified ratio based on counting statistics.

\section{CONCLUSION}

EUV TOF has unique properties that allow for nanoscale mass spectral imaging, such as mapping isotopic heterogeneity at the nanoscale. We have shown here that EUV TOF can map the ${ }^{235} \mathrm{U} /{ }^{238} \mathrm{U}$ isotope ratio in $100 \mathrm{~nm}$ and $1 \mu \mathrm{m}$ pixels, where more heterogeneity is revealed at the $100 \mathrm{~nm}$ spatial scale. Within the scope of this study, EUV TOF shows similar mapping capabilities to NanoSIMS at the nanoscale. Both EUV TOF and NanoSIMS detected statistically significant U isotopic heterogeneity, identifying microscale heterogeneity that was overlooked at larger spatial scales. We have also shown that EUV TOF can accurately measure the isotope ratio in silver from individual laser shots. EUV TOF is a good candidate for isotope ratio analyses and elemental/isotopic mapping at high spatial scales in fields such as nuclear forensics, geology, and biology.

\section{REFERENCES}

[1] Kompauer, M., Heiles, S. and Spengler, B., "Atmospheric Pressure MALDI Mass Spectrometry Imaging of Tissues and Cells at 1.4- $\mu \mathrm{m}$ Lateral Resolution," Nat. Methods 14, 90-96 (2017).

[2] Wang, J., Wang, Z., Liu, F., Cai, L., Pan, J.-B., Li, Z., Zhang, S., Chen, H.-Y., Zhang, X. and Mo, Y., "Vacuum Ultraviolet Laser Desorption/Ionization Mass Spectrometry Imaging of Single Cells with Submicron Craters," Anal. Chem. 90, 10009-10015 (2018).

[3] Nunez, J., Renslow, R., Cliff, J. B. and Anderton, C. R., "NanoSIMS for Biological Applications: Current Practices and Analyses," Biointerphases 13, 03B301-1 - 03B301-26 (2017).

[4] Gundlach-Graham, A., Burger, M., Allner, S., Schwarz, G., Wang, H. A. O., Gyr, L., Grolimund, D., Hattendorf, B. and Detlef, G., "High-Speed, High-Resolution, Multielemental Laser Ablation-Inductively Coupled Plasma-Time-ofFlight Mass Spectrometry Imaging: Part I. Instrumentation and Two-Dimensional Imaging of Geological Samples," Anal. Chem. 87, 8250-8258 (2015).

[5] Pan, Y., Hu, L. and Zhao, T., "Applications of Chemical Imaging Techniques in Paleontology," Natl. Sci. Rev. 6, 1040-1053 (2019).

[6] Kips, R., Weber, P. K., Kristo, M. J., Jacobsen, B. and Ramon, E., "Microscale Isotopic Variation Observed in Uranium Fuel Pellets with Implications for Nuclear Forensics," Anal. Chem. 91, 11598-11605 (2019).

[7] Reilly, D. D., Beck, C. L., Buck, E. C., Cliff, J. B., Duffin, A. M., Lach, T. G., Liezers, M., Springer, K. W. E., Tedrow, S. J. and Zimmer, M. M., "Focused Ion Beam for Improved Spatially-Resolved Mass Spectrometry and Analysis of Radioactive Materials for Uranium Isotopic Analysis," Talanta 211, 1-8 (2020).

[8] Fallon, C. M., Bower, W. R., Lyon, I. C., Livens, F. R., Thompson, P., Higginson, M., Collins, J., Heath, S. L. and Law, G. T. W., "Isotopic and Compositional Variations in Single Nuclear Fuel Pellet Particles Analyzed by Nanoscale Secondary Ion Mass Spectrometry," ACS Omega 5, 296-303 (2019).

[9] Varga, Z., Wallenius, M., Nicholl, A. and Mayer, K., "Assessment of Uranium Inhomogeneity and Isotope Imaging for Nuclear Forensics," Spectrochim. Acta B 171, 1-7 (2020).

[10] Krachler, M., Varga, Z., Nicholl, A., Wallenius, M. and Mayer, K., "Spatial Distribution of Uranium Isotopes in Solid Nuclear Materials Using Laser Ablation Multi-Collector ICP-MS," Microchem. J. 140, 24-30 (2018).

[11] Duffin, A. M., Springer, K. W., Ward, J. D., Jarman, K. D., Robinson, J. W., Endres, M. C., Hart, G. L., Gonzalez, J. J., Oropeza, D., Russo, R. E., Willingham, D. G., Naes, B. E., Fahey, A. J. and Eiden, G. C., "Femtosecond Laser Ablation Multicollector ICPMS Analysis of Uranium Isotopes in NIST Glass," J. Anal. At. Spectrom. 30, 1100-1107 (2015).

[12] Kuznetsov, I., Filevich, J., Dong, F., Woolston, M., Chao, W., Anderson, E. H., Bernstein, E. R., Crick, D. C., Rocca, J. J. and Menoni, C. S., "Three-Dimensional Nanoscale Molecular Imaging by Extreme Ultraviolet Laser Ablation Mass Spectrometry," Nat. Commun. 6, 1-6 (2015). 
[13] Green, T., Kuznetsov, I., Willingham, D., Naes, B. E., Eiden, G. C., Zhu, Z., Chao, W., Rocca, J. J., Menoni, C. S. and Duffin, A. M., "Characterization of Extreme Ultraviolet Laser Ablation Mass Spectrometry for Actinide Trace Analysis and Nanoscale Isotopic Imaging," J. Anal. At. Spectrom. 32, 1092-1100 (2017).

[14] Rush, L. A., Cliff, J. B., Reilly, D. D., Duffin, A. M. and Menoni, C. S., "Isotopic Heterogeneity Imaged in a Uranium Fuel Pellet with Extreme Ultraviolet Laser Ablation and Ionization Time-of-Flight Mass Spectrometry," Anal. Chem. 93, 1016-1024 (2021).

[15] Azov, V. A., Mueller, L. and Makarov, A. A., "Laser Ionization Mass Spectrometry at 55: Quo Vadis?," Mass Spectrom. Rev., 1-52 (2020).

[16] Rocca, J. J., Shlyaptsev, V., Tomasel, F. G., Cortazar, O. D., Hartshorn, D. and Chilla, J. L. A., "Demonstration of a Discharge Pumped Table-Top Soft-X-Ray Laser," Phys. Rev. Lett. 73, 2192-2195 (1994).

[17] Heinbuch, S., Grisham, M., Martz, D. and Rocca, J. J., "Demonstration of a Desk-Top Size High Repetition Rate Soft X-Ray Laser," Opt. Express 13, 4050-4055 (2005).

[18] Vaschenko, G., Etxarri, A. G., Menoni, C. S., Rocca, J. J., Hemberg, O., Bloom, S., Chao, W., Anderson, E. H., Attwood, D. T., Lu, Y. and Parkinson, B., "Nanometer-Scale Ablation with a Table-Top Soft X-Ray Laser," Opt. Lett. 31, 3615-3617 (2006).

[19] Rossall, A. K., Aslanyan, V., Tallents, G. J., Kuznetsov, I., Rocca, J. J. and Menoni, C. S., "Ablation of Submicrometer Holes Using an Extreme-Ultraviolet Laser," Phys. Rev. Applied 3, 064013 (2015).

[20] Berrill, M., Brizuela, F., Langdon, B., Bravo, H., Menoni, C. S. and Rocca, J. J., "Warm Photoionized Plasmas Created by Soft-X-Ray Laser Irradiation of Solid Targets," J. Opt. Soc. Am. B 25, B32-B38 (2008).

[21] Anderson, E. H., "Specialized Electron Beam Nanolithography for EUV and X-Ray Diffractive Optics," IEEE J. Quantum Electron. 42, 27-35 (2006).

[22] Schwantes, J. M., Marsden, O. and Pellegrini, K. L., "State of Practice and Emerging Application of Analytical Techniques of Nuclear Forensic Analysis: Highlights from the 4th Collaborative Materials Exercise of the Nuclear Forensics International Technical Working Group (ITWG)," J. Radioanal. Nucl. Chem. 311, 1441-1452 (2017).

[23] Mathew, K., Mason, P., Voeks, A. and Narayanan, U., "Uranium Isotope Abundance Ratios in Natural Uranium Metal Certified Reference Material 112-A," Int. J. Mass Spectrom. 315, 8-14 (2012).

[24] Zirakparvar, N. A., Hexel, C. R., Miskowiec, A. J., Smith, J. B., Ambrogio, M. W., Duckworth, D. C., Kapsimalis, R. and Ticknor, B. W., "A Nanosims $50 \mathrm{~L}$ Investigation into Improving the Precision and Accuracy of the ${ }^{235} \mathrm{U} /{ }^{238} \mathrm{U}$ Ratio Determination by Using the Molecular ${ }^{235} \mathrm{U}^{16} \mathrm{O}$ and ${ }^{238} \mathrm{U}^{16} \mathrm{O}$ Secondary Ions," Minerals 9, 307-329 (2019).

[25] Wiesendanger, R., Grimaudo, V., Tulej, M., Riedo, A., Lukmanov, R., Ligterink, N., Fausch, R., Shea, H. and Wurz, P., "The LMS-GT Instrument - A New Perspective for Quantification with the LIMS-TOF Measurement Technique," J. Anal. At. Spectrom. 34, 2061-2073 (2019). 